\title{
Field performance assessment versus asphalt pavement design considerations
}

\author{
A. Loizos, K. Gkyrtis, C. Plati \& K. Georgouli \\ Laboratory of Pavement Engineering, National Technical University of Athens (NTUA), Athens, Greece
}

\begin{abstract}
Knowledge of pavement structural condition is desirable throughout a pavement's service life. Although the as-built pavement condition in terms of bearing capacity is most critical for the evolution of its future performance, it still remains challenging for pavement engineers to investigate any dependencies of the field assessment process on the pavement design. The latter becomes even more significant when the assumptions and loading principles used for material characterization during the design, differ from those that can be implemented in the field through the common utilization of Non-Destructive Testing (NDT) systems, including among others, the Falling Weight Deflectometer (FWD). On these grounds, the present research study considers field evaluation challenges of a new asphalt pavement structure of a heavy-duty motorway designed according to a robust international analytical design method. In particular, standardized Asphalt Concrete (AC) materials were assumed for the asphalt base and binder courses, whose stiffness characteristics were determined for the design analysis through the two-point bending test on trapezoidal specimens. Contrariwise, NDT testing was performed in the field and cylindrical cores were also extracted to assess material performance in the laboratory through the uniaxial compression test mode. The analysis for the pavement evaluation follows mechanistic perspectives demonstrating how to address potential limitations of pavement structural evaluation.
\end{abstract}

Keywords: Asphalt pavements, zero-point assessment, viscoelastic materials, pavement evaluation

\section{INTRODUCTION}

Pavement condition assessment at any time t throughout a pavement's lifespan is more than desirable, as it enables rational scheduling of maintenance actions (Plati et al. 2020a). In particular, the assessment shortly after construction or else during $t=0$, which will be hereinafter referred to as "zero-point" assessment, is of utmost significance as its purpose is twofold. First, it provides information on the condition that was achieved during construction (quality control) and second, it serves as historic data (Georgouli and Loizos 2017). While the goal of the former is to define whether the constructed pavement meets the pavement and material design principles, the latter enables the establishment of a reference status based on the asbuilt pavement condition. This aspect is rather useful for future performance monitoring during a pavement's service life.

For both of the afore-mentioned purposes during pavement evaluation, the major engineering challenge lies upon using tools and analysis theories that lead to reliable results and conclusions in respect to pavement condition. Commonly, data collected through Non-Destructive Testing (NDT) systems, including the Falling Weight Deflectometer (FWD) and the Ground 
Penetrating Radar (GPR), is used as input to back-calculate the pavement stiffness profile and proceed with a response analysis that enables pavement life expectancy estimation (Plati et al. 2020b). The Multi-Layered Elastic Theory (MLET) is most often used as the background theory of the previous analysis steps (Marecos et al. 2017, Crook et al. 2012).

However, there is enough evidence that the Asphalt Concrete (AC) materials exhibit viscoelastic behavior, since they are temperature- and loading rate- dependent (Grellet et al. 2012, Chabot et al. 2010). Viscoleastic material characterization can be performed either through laboratory tests on field cores (Gkyrtis et al. 2021, Georgouli et al. 2015) or through the use of algorithms that estimate the AC dynamic modulus $E^{*}$ (Georgouli and Loizos 2017). No matter the way followed, the use of different approaches to analyze the pavement structure (i.e. through elastic or viscoelastic calculations) can lead to discrepancies on its bearing capacity and life expectancy estimation with a profound effect on maintenance decision-making issues.

On this context, although the as-built pavement condition in terms of bearing capacity is most critical for the evolution of its future performance, it still remains challenging for pavement engineers to investigate any dependencies of the field assessment process on the pavement design. Focusing on the zero-point condition, the latter becomes even more significant because the assumptions and loading principles used for material characterization during the design may differ from those that can be implemented in the field through the use of NDTs (Gkyrtis et al. 2021).

This is the case of the experimental pavement selected in this study for the investigation purposes. In particular, the selected pavement was designed according to an international analytical and robust design method (LCPC-SETRA, 1997). Standardized AC materials were assumed for the asphalt base and binder courses, whose stiffness characteristics were determined for the design analysis through the Two-Point Bending (2PB) test on trapezoidal specimens in the laboratory. The latter implies that the design considerations are not compatible with the cylindrical cores that are usually extracted in the field. Moreover, the FWD load imposed in-situ through a segmented loading plate is not directly comparable with the design loading principles. Such issues make even more complex the zero-point condition assessment in new pavements, since dependencies on the design process may exist. In addition, material characterization in the Linear Visco-Elastic (LVE) region requires core extraction and sophisticated laboratory equipment to obtain the $E^{*}$, aspects that usually make it tedious for pavement engineers to consider advanced material characterization in the field.

Nevertheless, pavement engineers are responsible for effectively incorporating pavement mechanistic principles into common engineering issues. The objective of this study is to investigate structural assessment challenges during the zero-point assessment and develop a simplified approach based on NDT and laboratory data in order to reach a reliable overview of the pavement condition. To meet the research aim, a field experiment was organized along an experimental asphalt pavement section, which is part of a heavy-duty motorway and belongs to the trans-European transport network. The analysis was based on response calculations and the emphasis was put on the tensile strains at the bottom of the AC layers.

\section{STRUCTURE, MATERIALS AND TESTING}

A typical cross section of the trial asphalt pavement is shown in Figure 1. At the time of the experiment, the AC layers included an asphalt binder and asphalt base layer with limestone aggregates. The aggregate gradation is given in Table 1. The asphalt material had a penetration grade ranging from $35-36 \mathrm{PEN}$ and a softening point of $55-56^{\circ} \mathrm{C}$. Base layer consisted of unbound granular material and the subgrade layer consisted of natural gravel. 


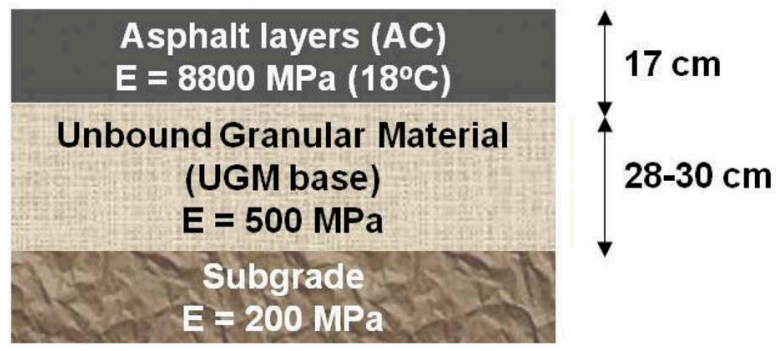

Figure 1. Typical pavement cross-section.

Table 1. Aggregate gradation for AC materials ( $\%$ passing).

\begin{tabular}{lll}
\hline Sieve $(\mathrm{mm})$ & Binder course & Base course \\
\hline 19 & 100 & 91 \\
9.5 & 78 & 65 \\
4.75 & 52 & 44 \\
0.075 & 4 & 3.4 \\
\hline
\end{tabular}

In respect to the experiment, a $50 \mathrm{kN}$ load was imposed by the FWD on the pavement surface at the outer wheel-path of the heavy-duty traffic lane. Surface deflections were recorded by nine sensors. Temperature measurements in the middle of the AC layers were performed through properly drilled holes within the pavement. At each of the investigated locations (P1P7), multiple FWD measurements were undertaken at the temperatures of Table 2 in order to obtain a better overview of AC temperature sensitivity. These multiple measurements were taken at different times of the day. GPR surveys and coring yielded granular base and AC layer thicknesses respectively. GPR signal analysis led to thickness prediction errors ranged from less than $1 \%$ up to $8 \%$ in accordance with previous studies (Plati et al. 2020b).

Table 2. Measured temperatures at the middle of AC layers.

\begin{tabular}{ll}
\hline Position & Temperature $\left({ }^{\circ} \mathrm{C}\right)$ \\
\hline P1 & $26.7,31.2,35.9$ \\
P2 & $27.0,31.5,36.2$ \\
P3 & $27.4,31.8,36.7$ \\
P4 & $24.0,39.7$ \\
P5 & $24.0,39.6$ \\
P6 & $24.0,39.6$ \\
P7 & $24.0,39.7$ \\
\hline
\end{tabular}

As per the laboratory testing, the dynamic modulus test (AASHTO, 2001) was performed on seven cores extracted in-situ at the same positions of FWD testing. $E^{*}$ was determined under the Uniaxial Compression (UC) test mode. In particular, thirty combinations of a controlled sinusoidal (haversine) compressive loading were imposed at each core, including five temperatures $\left(4,15,20,25\right.$ and $\left.37^{\circ} \mathrm{C}\right)$ and six frequencies $(25,10,5,1,0.5$ and $0.1 \mathrm{~Hz})$. Difficulties in performing the dynamic modulus test at -10 and $54^{\circ} \mathrm{C}$ led to the selection of the previous temperatures. Similar problems have been also mentioned elsewhere (Bennert and Williams 2009). 


\section{ANALYSIS CONCEPTUAL FRAMEWORK}

Two kinds of response calculations were performed: (a) viscoelastic analysis based on the laboratory determined $E^{*}$ master curves of field cores, and (b) viscoelastic analysis based on the theoretical $E^{*}$ master curve of the standardized material assumed in the design. The first case represented the as-built pavement status. For comparison purpose, the second case generated an additional reference strain state adjusted to the field conditions. This implies that the AC characteristics constituted the only factor that could differentiate the pavement condition between the "expected" behavior (in accordance with pavement design considerations) and the "as-built" behavior. The analysis concept is illustrated in Figure 2. The stiffness profile of the underlying layers, pavement geometry, loading conditions and temperature were considered identical in both cases in respect to the in-situ evaluation process. In particular, base and subgrade layers were assumed to consist of elastic materials and their weighted moduli were considered equal to 500 and $200 \mathrm{MPa}$ respectively based on FWD results at all locations.

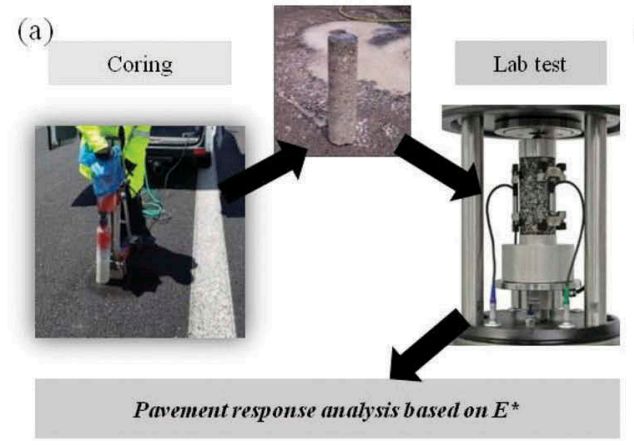

(b)

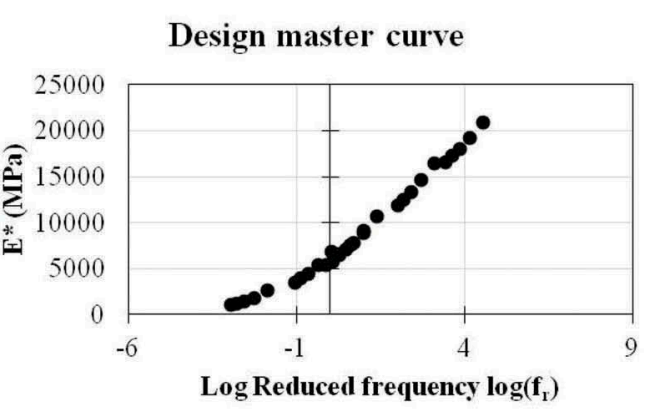

Figure 2. Analysis framework for the (a) "as-built" condition, and the (b) "expected" condition.

The adoption of the strain analysis approach dealt with potential incompatibilities on AC moduli assessment stemming from the different laboratory tests utilized for AC characterization. Furthermore, during the design process, an axle load of $130 \mathrm{kN}$ with dual tires was assumed, which generates a strain condition not directly comparable with field related conditions. As such, the developed framework with the FWD loading for both analysis cases can overcome potential obstacles in regards to the dependency of pavement assessment on the design principles.

For both analysis cases (a) and (b), the pavement response analysis was performed in the ViscoRoute software (Chabot et al. 2010), through the calibration of the rheological HuetSayegh model. This model consists of two branches including a spring and two parabolic dampers in the first branch and a spring in the second branch (Figure 3).

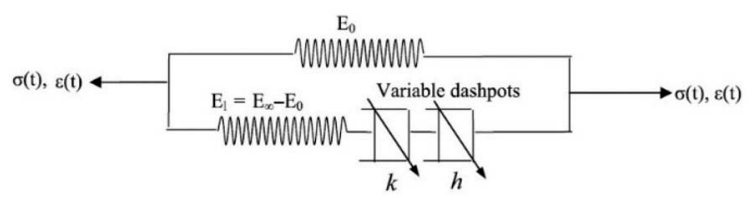

Figure 3. Representation of the Huet-Sayegh model.

Its mathematical formulation is:

$$
E(\omega, \theta)=E_{0}+\frac{E_{\propto}-E_{0}}{1+\delta(j \omega \tau(\theta))^{-k}+(j \omega \tau(\theta))^{-h}}
$$


where $E_{\infty}$ is the instantaneous elastic modulus $(\mathrm{MPa}), E_{0}$ is the static elastic modulus (MPa), $k$ and $h$ are dimensionless exponents of the parabolic dampers, and $\delta$ is a positive dimensionless coefficient. Also, $\omega=2 \pi f$ ( $f=$ loading frequency) and $\theta=$ temperature. The temperature function $\tau(\theta)$ is defined as per Eq. $2\left(A_{0}, A_{1}, A_{2}=\right.$ thermal coefficients):

$$
\tau(\theta)=\exp \left(A_{0}+A_{1} \theta+A_{2} \theta^{2}\right)
$$

The calibration of Eq. 1 was based on nonlinear least squares regression with the minimization of the Standard-Square Error (SSE), defined as:

$$
S S E=\sum_{i=1}^{n}\left(\log E_{l a b}^{*}-\log E_{H S}^{*}\right)^{2}
$$

where $E^{*}{ }_{l a b}$ is the experimental dynamic modulus $(\mathrm{MPa})$ and $\mathrm{E}^{*}{ }_{H S}$ is the predicted dynamic modulus (MPa). It is noted that the SSE ranged satisfactorily from 0.005-0.028 for all cores, indicating a good adaptability of the Huet-Sayegh model to $E^{*}$ data. Thereafter, the response calculations were performed for the FWD load level of $50 \mathrm{kN}$ (tire pressure $707 \mathrm{kPa}$ ) considering all of the temperatures enlisted in Table 2 and a moving speed of $80 \mathrm{~km} / \mathrm{h}$. FWD frequency was estimated equal to $18 \mathrm{~Hz}$ according to a methodology presented in Crow (1998) and was thereafter transformed to the previous speed according to an equation presented in Mollenhauer et al. (2009).

Finally, considering the integrity destruction in new pavements due to the coring process, the ability of FWD measured deflections to predict the as-built viscoelastic strains was investigated through statistical correlation. The rationale behind this approach was also strengthened because of the highway pressing constraints against lane closures, which is a critical issue for the case of in-service pavements.

\section{RESULTS}

\subsection{Overview of viscoelastic response analysis}

The response analysis with the design master curve as input led to an average longitudinal strain $\varepsilon_{\mathrm{x} \text {-ref }}=71.3 \mu \mathrm{m} / \mathrm{m}$ and an average transverse strain $\varepsilon_{\mathrm{y}-\mathrm{ref}}=80.4 \mu \mathrm{m} / \mathrm{m}$ at the reference temperature of $18^{\circ} \mathrm{C}$ (assumed in the design), with a coefficient of variation equal to $3.7 \%$ and $3.6 \%$ respectively. For engineering analysis purposes, the above strains were assumed as the reference strain status for the pavement assessment at the same temperature. As such, in Figure 4, viscoelastic strains based on the $E^{*}$ of field cores are presented together with the reference strains; the solid line corresponds to the $\varepsilon_{\mathrm{x}-\mathrm{ref}}=71.3 \mu \mathrm{m} / \mathrm{m}$ and the dashed line corresponds to the $\varepsilon_{\mathrm{y}-\mathrm{ref}}=80.4 \mu \mathrm{m} / \mathrm{m}$.

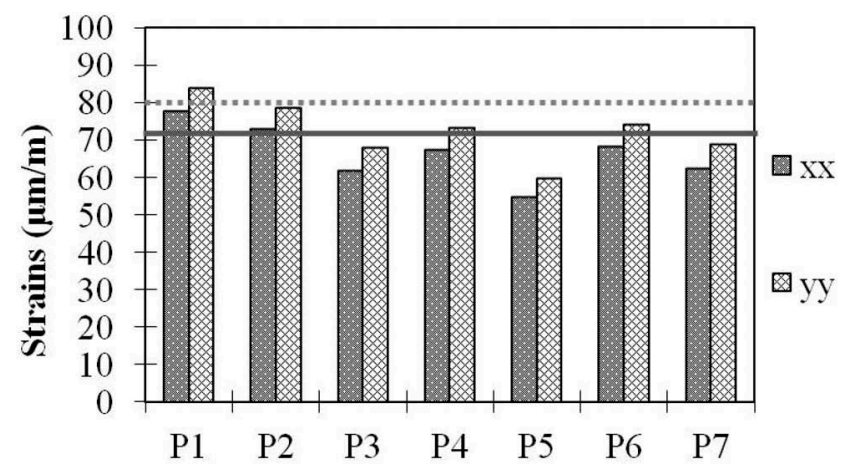

Figure 4. Viscoelastic strains at $18^{\circ} \mathrm{C}$. 
It can be seen, that 5 out of 7 locations exhibit lower strains at the bottom of $\mathrm{AC}$ in respect to the expected strains (solid and dashed lines). The maximum difference in the cases where strains from the as-built condition exceed the reference values (locations P1-P2) is 6.8 and $4.5 \mu \mathrm{m} / \mathrm{m}$ for the longitudinal and transverse strains respectively. In order to investigate whether this trend is consistent or not for the whole spectrum of measured temperatures (Table 2), the strain comparison between the expected and the as-built condition was repeated considering the variable field temperatures at each location (Figure 5). It can be seen that for all the measured temperatures, the expected strains exceed the strains calculated considering the as-built material condition.
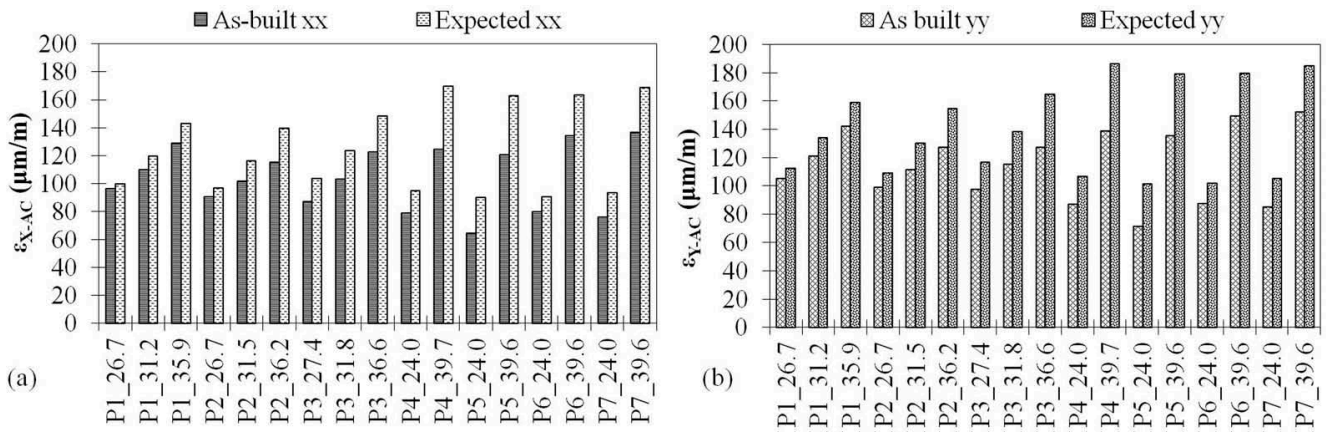

Figure 5. Comparison of viscoelastic strains (a) longitudinally and (b) transversely.

The magnitude of differences varies depending on the analysis temperature, so the impact of AC temperature on pavement evaluation is highlighted. In particular, averaged strains are presented in Figure 6 considering all the coring locations and similar field temperatures. Indeed, the expected strains exhibit a more abrupt change against temperature increase, which may imply that the material placed in-situ was less sensitive to the temperature increase as opposed to the expected performance of the standardized material that was assumed in the design.

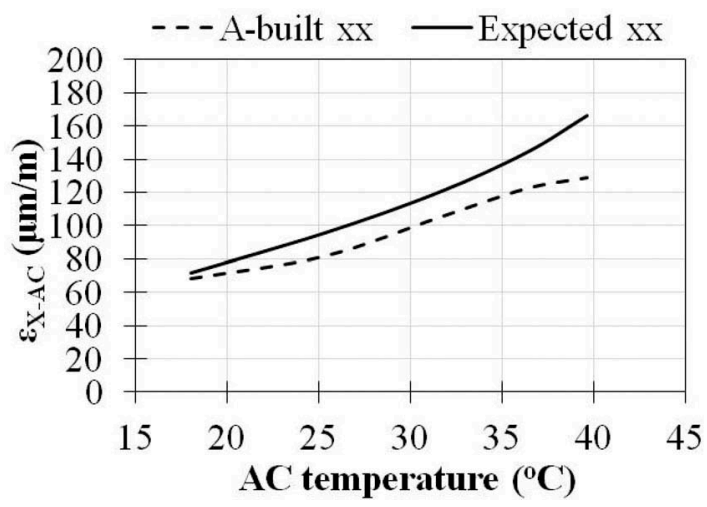

Figure 6. Longitudinal strain sensitivity against temperature.

Additionally, the material sensitivity on temperature is also profound in Figure 7, considering the full longitudinal and transverse strain profiles indicatively for location $\mathrm{P} 4$ at the highest measured temperature of $39.7^{\circ} \mathrm{C}$. The asymmetry around the loading point in the longitudinal strain profile is less obvious at the as-built strain profile. Similarly, a slight asymmetry is also observed transversely on the expected strain profile around the peak value, which is less visible on the as-built strain profile. The previous remarks indicate again that the 
AC behavior of the material placed in-situ is less prone to temperature increase than the behavior of the standardized material considered in the design.
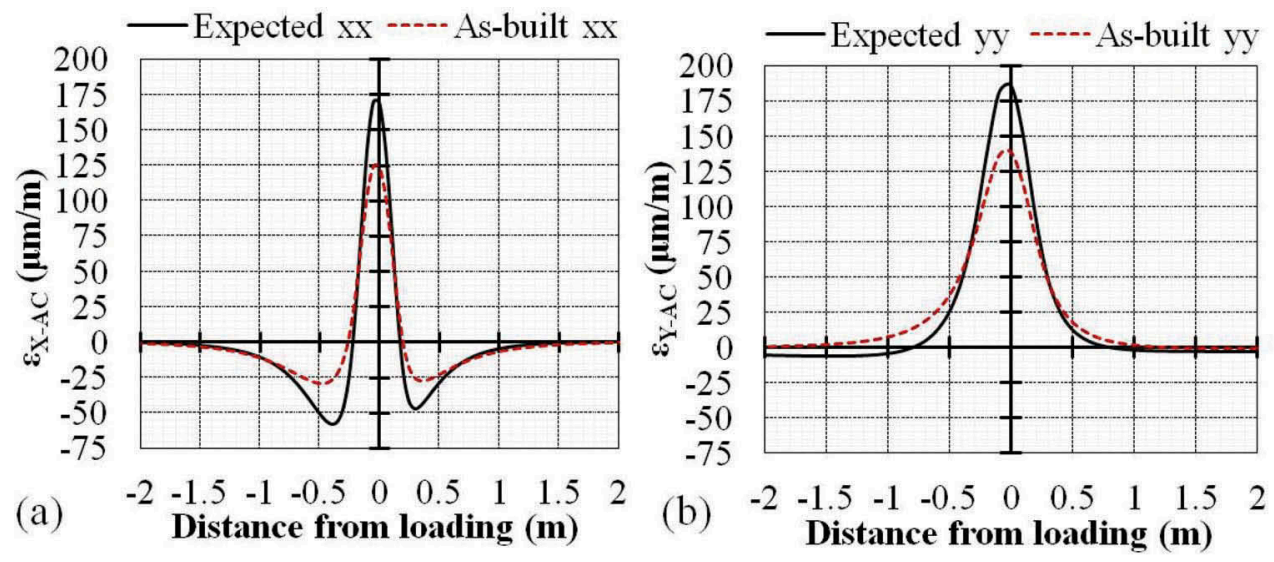

Figure 7. Indicative strain profiles at $39.7^{\circ} \mathrm{C}$ (a) longitudinally, and (b) transversely.

Overall, the proposed strain analysis approach effectively demonstrated the material behavior differentiation when moving from the design process to the field, implying that coring process might be needed even during the assessment at $\mathrm{t}=0$, since the existence of properly calibrated algorithms for the non-destructive prediction of $E^{*}$ is usually a matter of concern. In addition, utilizing design $E^{*}$ data, even at $\mathrm{t}=0$, could result in erroneous estimations of the pavement bearing capacity estimation.

\subsection{Correlations between NDT data and viscoelastic strains}

At this stage, the ability of the FWD measured deflections to act as potential predictors of the viscoelastic strains was investigated. From a linear regression analysis, a promising potential was found, since both longitudinal and transverse strains correlated well with the AC temperature and the Surface Curvature Index $(S C I)$, defined as the difference of the surface deflections $d_{0}$ and $d_{300}\left(S C I=d_{0^{-}} d_{300}\right)$. These deflections are measured at a distance of 0 and $300 \mathrm{~mm}$ away from the centre of the FWD loading plate. The longitudinal strain variation against AC temperatures and SCI is shown in Figure 8. As can be seen, temperature appears to have better prediction ability than SCI. Nevertheless, it was decided to combine both parameters in order to assess the feasibility of strain estimation.

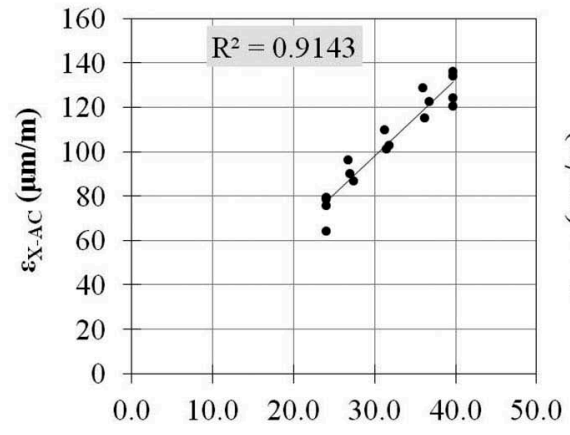

(a)
AC temperature $\left({ }^{\circ} \mathrm{C}\right)$

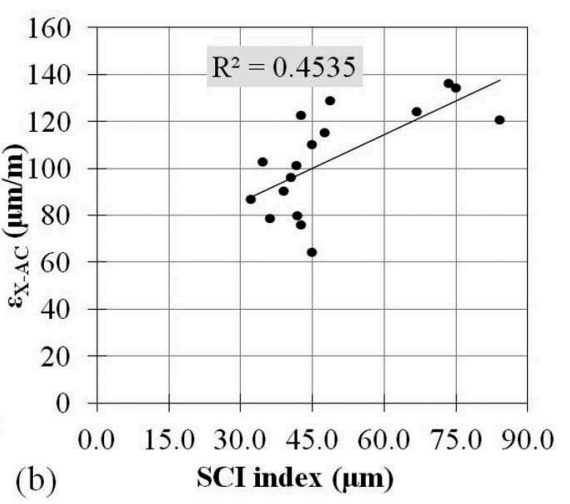

(b)

Figure 8. Correlations between (a) strains and AC temperature, and (b) strains and SCI. 
As such, moving forward with the modelling approach presented in Loizos et al. (2020), the linear regression followed in this study resulted in a typical equation: $\varepsilon_{x-A C \text { predicted }}=a \cdot T+b \cdot S C I+c$, where $a, b$ and $c$ are regression constants. The $R^{2}$ value of the equation fit was found to be 0.91 . The regression constants are not intentionally given, since only data from a unique structure was used, thus the use of the equation cannot be replicated elsewhere. Nevertheless, the presented approach might be proved beneficial in case the coring process needs to limited.

\section{CONCLUDING REMARKS}

This study demonstrated a simplified approach to perform structural evaluation of a new asphalt pavement by following mechanistic perspectives. The proposed approach could be used to overcome potential dependencies of the pavement evaluation process on pavement design principles. This was the case of the investigated pavement, as the standardized material assumed in the design was theoretically characterized through a test mode that is not compatible with the cylindrical cores that are usually extracted in the field.

Although the experiment took place at $\mathrm{t}=0$, significant differentiation of the $\mathrm{AC}$ material was observed during the viscoelastic analysis. The assessment followed a strain comparison concept instead of moduli assessment, since moduli are in general expected to be noncomparable due to the different laboratory methods for the estimation of $E^{*}$. Moving forward with the investigation and based on the good variety and availability of NDT data, a practical framework was shown that could assist real-scale pavement evaluation and the related calculations, by integrating mainly NDT data and limited coring data (needed for model calibration) in order to quickly estimate viscoelastic responses.

In other words, pavement assessment at $\mathrm{t}=0$ could be potentially addressed by estimating field viscoelastic strains through NDT data and limited coring (representing the as-built strain status) and simultaneously using the design modulus master curve to generate a reference response status adjusted at field conditions (to reflect the expected strain status according to pavement design).

To conclude, this paper contributed on how to deal with potential pavement structural evaluation limitations during the zero-point assessment and highlighted the significance of this assessment. No matter how well or not a pavement has been designed and constructed, this is expected to be serviceable for a long period beyond its design life. As such, formulating a method for the as-built pavement condition assessment contributes to a database development in order to systematically monitor pavement performance in the future and make a rational scheduling of potential maintenance or rehabilitation actions in the long term. Nevertheless, additional research is needed with a wider material spectrum and NDT data to further assess the feasibility of the proposed approach.

\section{REFERENCES}

AASHTO T342-11 (2001) Standard method of test for determining dynamic modulus of HMA. American Association of State Highway and Transportation Officials, Washington.

Bennert, T. and Williams, S.G., 2009. Precision of AASHTO TP62-07 for use in mechanistic-empirical pavement design guide for flexible pavements. Transportation Research Record: Journal of the Transportation Research Board, 2127: 115-126.

Chabot, A., Chupin, O., Deloffre, L. and Duhamel, D., 2010. ViscoRoute 2.0: a tool for the simulation of moving load effects on asphalt pavement. Road Materials and Pavement Design, 11(2): 227-250.

Crook, A.L., Montgomery, S.R. and Guthrie, W.S., 2012. Use of falling weight deflectometer data for network-level flexible pavement management. Transportation Research Record: Journal of the Transportation Research Board, 2304: 75-85.

CROW record, Deflection profile-not a pitfall anymore. Survey and interpretation methodology-falling weight deflection measurements. Crow, EDE, Netherlands, 1998.

Georgouli, K. and Loizos, A., 2017. E* prediction algorithm for pavement quality control assessment. Loizos et al. (Eds): Proceedings of the $10^{\text {th }}$ International Conference on the Bearing Capacity of 
Roads, Railways and Airfields (BCRRA 2017, June 28-30, Athens, Greece), Taylor \& Francis group: 799-805.

Georgouli, K., Pomoni, M., Cliatt, B. and Loizos A. 2015. A simplified approach for the estimation of HMA dynamic modulus for in service pavements. In Proceedings of the $6^{\text {th }}$ International Conference on Bituminous Mixtures and Pavements (ICONFBMP), June 10-12, 2015, Thessaloniki, Greece, Taylor and Francis Group, pg. 661-670.

Gkyrtis, K., Loizos, A. and Plati, C. 2021. A mechanistic framework for field response assessment of asphalt pavements. International Journal of Pavement Research and Technology, 14, 174-185.

Grellet, D., Dore, G., Kerzreho, J.P., Piau, J.M., Chabot, A. and Hornych, P., 2012. Experimental and theoretical investigation of three dimensional strain occurring near the surface in asphalt concrete layers. In Proceedings of the $7^{\text {th }}$ Rilem International Conference on Cracking in Pavements, France, SPRINGER: $1017-1027$.

LCPC-SETRA, French Design Manual for Pavement Structures: Guide Technique, 1997.

Loizos, A., Gkyrtis, K. and Plati, C., 2020. Modelling asphalt pavement responses based on field and laboratory data. Chabot et al. (Eds): Accelerated Pavement Testing to Transport Infrastructure Innovation, LNCE 96: 438-447.

Marecos, V., Fontul. S., Antunes, M.L. and Solla, M., 2017. Evaluation of a highway pavement using non-destructive tests: Falling Weight Deflectometer and Ground Penetrating Radar. Construction and Building Materials, 154: 1164-1172.

Mollenhauer, K., Wistuba, M. and Rabe, R., 2009. Loading Frequency and Fatigue: In situ conditions \& Impact on Test Results. $2^{\text {nd }}$ Workshop on Four Point Bending, Pais (ed.), University of Minho, Portugal, pp. 261-276.

Plati, C., Loizos, A. and Gkyrtis, K. 2020a. Assessment of modern roadways using non-destructive geophysical surveying techniques. Surveys in Geophysics, 41, 395-430.

Plati, C., Gkyrtis, K. and Loizos, A., 2020b. Integrating non-destructive testing data to produce asphalt pavement critical strains. Nondestructive Testing and Evaluation, https://doi.org/10.1080/105897 59.2020 .1834555 . 